\title{
BMJ Open Minimising post-operative risk using a Post-Anaesthetic Care Tool (PACT): protocol for a prospective observational study and cost-effectiveness analysis
}

\author{
Maryann Street, ${ }^{1,2}$ Nicole M Phillips, ${ }^{1}$ Bridie Kent, ${ }^{3}$ Stephen Colgan, ${ }^{4}$ \\ Mohammadreza Mohebbi ${ }^{5}$
}

To cite: Street M,

Phillips NM, Kent B, et al. Minimising post-operative risk using a Post-Anaesthetic Care Tool (PACT): protocol for a prospective observational study and costeffectiveness analysis. BMJ Open 2015;5:e007200. doi:10.1136/bmjopen-2014007200

- Prepublication history for this paper is available online. To view these files please visit the journal online (http://dx.doi.org/10.1136/ bmjopen-2014-007200).

Received 13 November 2014 Revised 9 March 2015 Accepted 12 March 2015

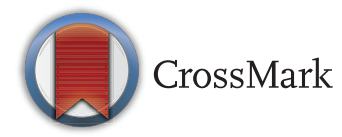

For numbered affiliations see end of article.

\section{Correspondence to} Dr Maryann Street; maryann.street@deakin. edu.au

\section{ABSTRACT}

Introduction: While the risk of adverse events following surgery has been identified, the impact of nursing care on early detection of these events is not well established.

A systematic review of the evidence and an expert consensus study in post-anaesthetic care identified essential criteria for nursing assessment of patient readiness for discharge from the post-anaesthetic care unit (PACU). These criteria were included in a new nursing assessment tool, the Post-Anaesthetic Care Tool (PACT), and incorporated into the post-anaesthetic documentation at a large health service. The aim of this study is to test the clinical reliability of the PACT and evaluate whether the use of PACT will (1) enhance the recognition and response to patients at risk of deterioration in PACU; (2) improve documentation for handover from PACU nurse to ward nurse; (3) result in improved patient outcomes and (4) reduce healthcare costs.

Methods and analysis: A prospective, nonrandomised, pre-implementation and postimplementation design comparing: (1) patients $(n=750)$ who have surgery prior to the implementation of the PACT and (2) patients ( $n=750)$ who have surgery after PACT. The study will examine the use of the tool through the observation of patient care and nursing handover. Patient outcomes and cost-effectiveness will be determined from health service data and medical record audit. Descriptive statistics will be used to describe the sample and compare the two patient groups (pre-intervention and post-intervention). Differences in patient outcomes between the two groups will be compared using the Cochran-Mantel-Haenszel test and regression analyses and reported as ORs with the corresponding $95 \% \mathrm{Cls}$.

Conclusions: This study will test the clinical reliability and cost-effectiveness of the PACT. It is hypothesised that the PACT will enable nurses to recognise and respond to patients at risk of deterioration, improve handover to ward nurses, improve patient outcomes, and reduce healthcare costs.

\section{INTRODUCTION}

Surgical care is an integral part of healthcare throughout the world, with an estimated 234

\section{Strengths and limitations of this study}

This study will be the first to examine the impact of nursing care in the immediate post-operative period on clinical risk, adverse events and postoperative complications.

- The prospective design and direct observation of nursing care within the post-anaesthetic care unit will identify processes and data not captured in the medical record, such as consultations with medical staff, nursing handover and gaps in documentation.

- There may be factors external to the implementation of Post-Anaesthetic Care Tool that may increase or decrease surgical risk, reducing the ability of this study to determine causality. However, it is a practical and effective design that has previously been used to demonstrate the benefit of a surgical safety checklist.

million operations performed annually. Studies in industrialised countries have shown a perioperative death rate from inpatient surgery of $0.4-0.8 \%$ and a rate of major complications of $3-17 \% .^{2-5}$ Approximately $40 \%$ of in-hospital complications are associated with surgery and $15 \%$ of surgical patients will experience at least one complication, ${ }^{6}$ with bleeding, cardiac and respiratory problems, and infection being the most commonly occurring events. ${ }^{7}$ Hospital costs for surgical patients experiencing a complication are substantially higher than for patients without a complication. ${ }^{8}$ For example, patients suffering pneumonia after surgery have a $55 \%$ increase in hospital costs and $89 \%$ increase in length of hospital stay. ${ }^{9}$

The time immediately following an operation or procedure is a critical period for the patient's recovery. The intensive observation of patients in the post-anaesthetic care unit (PACU) by nurses can result in the early detection of complications and adverse 
events. ${ }^{10}$ These complications and adverse events include clinical deterioration, uncontrolled pain, unplanned admission to intensive care unit (ICU), prolonged hospital stay, disability or death. Techniquerelated complications, wound infections, and post-operative bleeding produced nearly half of all surgical adverse events. ${ }^{3}$ The early recognition of deterioration and the starting of therapy in PACU can prevent these complications or reduce their severity.

Importantly, during transitions of care, handover is key to providing safe patient care. It has been shown that any errors in communication can compromise patient safety, and may increase staff frustration due to inefficiency, delays and an increase in workload. ${ }^{11}$ Nurses can face a dilemma about the right time to transfer patients from PACU to general wards. ${ }^{12}$ There has been a trend towards the use of objective scoring systems to help nurses assess when a patient is ready to go back to the ward or be discharged to home following day surgery. ${ }^{13}{ }^{14}$ A systematic review identified there is limited evidence with regard to the criteria that are the essential components of these systems, such as the assessment of conscious state, pain, blood pressure, nausea and vomiting. ${ }^{15} 16$

Following the systematic review, an international expert consensus study, comprising anaesthetists and perioperative nurses, was conducted. ${ }^{17}$ Based on the findings of these two studies, our team identified essential criteria for the nursing assessment of patient readiness for discharge from the PACU, but the relationship of these essential criteria to safe patient discharge was yet to be evaluated.

\section{AIMS}

The research question for this study is: "Will the PACT, developed from the evidence and expert consensus, result in improved patient outcomes and reduced health care costs?" The aim is to evaluate whether the use of Post-Anaesthetic Care Tool (PACT), for nursing assessment of patient readiness for discharge following surgery, would (1) enhance the recognition and response to patients at risk of deterioration in PACU; (2) improve documentation for handover from PACU nurse to ward nurse, (3) result in improved patient outcomes and (4) reduce healthcare costs.

\section{METHODS AND ANALYSIS}

\section{Study design}

This pre-implementation and post-implementation study will use a prospective non-randomised design with (1) a group who have surgery prior to the introduction of the PACT and (2) a group who have surgery at least 3 months after the introduction of the PACT. The study is an observational study to evaluate the possible effect of a change in nursing practice in PACU, where randomisation of patients to a treatment group or a control group was outside the control of the researchers. Furthermore, randomisation within the hospitals included in the study would have been impractical.
Identification of similar hospitals as control hospitals would not have produced reliable data as those control hospitals would have been from a different health service with different policies and procedures.

\section{Setting}

The study will be conducted in one health service using two convenience samples of consecutive adult patients, admitted for any elective surgical procedure that results in a transfer to PACU. The health service has three large metropolitan acute care hospitals that have approximately 13000 elective surgical admissions per year.

The health service was chosen as the three hospitals varied in the type and complexity of surgery performed, acuity of patients and process of discharge from PACU. This ensures that the PACT will be evaluated in a variety of situations. All three hospitals conduct elective and emergency surgical procedures. Hospital A is an outer metropolitan community hospital with 180 beds, while hospitals $\mathrm{B}$ and $\mathrm{C}$ are located closer to the city of Melbourne with approximately 400 and 280 beds, respectively. Hospitals B and C both have ICUs, while hospital A does not. If a patient needs admission to ICU following surgery at hospital A, this requires an interhospital transfer with an associated increase in cost to the health service. Hospitals A and B discharge patients from PACU to a ward or day procedure unit with nursing handover occurring in PACU. In contrast, in hospital $\mathrm{C}$ patients are transferred by PACU nurses to the ward or day procedure unit, where nursing handover occurs.

\section{Participants}

The study population will comprise nurses working in PACU and surgical patients having a procedure during the study period.

Inclusion criteria (patients):

- Aged 18 years or older,

- Have undergone elective surgery and have been admitted to PACU.

Exclusion criteria (patients):

- Have undergone an emergency procedure;

- Admitted for a minor procedure that only requires sedation;

- Planned ICU admission from theatre or PACU.

\section{Sample size}

The primary outcome is the rate of adverse events. Given the diverse demographic characteristics of patients across the three hospitals, we expect our sample to be similar in demographic characteristics to the Australian population admitted to public hospitals for elective surgical procedures. Sample size calculations were based on an adverse event incidence of $12 \%$. This rate was determined from review of patient medical records at the three hospitals and the results of published studies, which have found that between $7.3 \%$ and $16.7 \%$ of surgical patients will experience an adverse 
event. ${ }^{6} 1819$ In order to detect a $7 \%$ difference between the (control vs intervention period) groups (ie, $12 \%$ vs $5 \%$ ), or an OR of at least 2.6, using a two-sided Cochran-Mantel-Haenszel $(\mathrm{CMH})$ test conducted at the $5 \%$ significance level with $80 \%$ power, 750 patients per group (250 before and 250 after the intervention at each of the three hospitals) are required in the study. Our sample size has been inflated to allow for a design effect of two accounting for within hospital clustering effect.

\section{The intervention}

The intervention in the study is a nursing assessment tool, the PACT, developed from the evidence obtained from a systematic review and expert consensus study. ${ }^{15-17}$ The PACT will be introduced at all three hospitals to standardise care across the health service. Nursing, medical, pharmacy and health information representatives from the health service were consulted to finalise the instrument prior to implementation in PACU. The PACT involves a track and trigger system for assessment of a patient's conscious state, vital signs (oxygen levels, respiration rate, blood pressure, temperature), symptoms (pain, nausea, vomiting), level of activity and care plan. It also contains a checklist of criteria that must be met prior to discharge from PACU (eg, last 2 sets of observations are not in Medical Emergency Team (MET)/modified MET criteria, no active vomiting, pain management

\section{Box 1 Complications and serious adverse events}

- Clinical deterioration*

- Code Blue or Medical Emergency Team Call

- Cardiac arrest

- Respiratory failure or failed extubation

- Cerebrovascular accident

- Development of neurological deficit not present on admission

- Excessive blood loss

- Pulmonary embolism

Uncontrolled pain

- Excessive nausea or vomiting

- Medication error or adverse drug reaction

- Discharge delay from post-anaesthetic care unit (PACU; greater than $2 \mathrm{~h}$ from time of admission to PACU)

- Unplanned return to operating theatre during this admission

- Unplanned intensive care or high-dependency unit admission

- Unplanned transfer to another hospital

- Readmission to hospital for a complication relating to the surgical admission

- Unexpected death (ie, not an expected outcome of the disease during hospitalisation)

- Prolonged length of hospital admission compared with the expected length for the clinical condition

*Notes: clinical deterioration determined through assessment of respiration (difficulty breathing, respiration rate less than 8 or greater than 30/min; oxygen saturation less than $90 \%$ despite oxygen therapy), circulation (heart rate less than 50 or more than $130 \mathrm{bpm}$, systolic blood pressure less than $90 \mathrm{~mm} \mathrm{Hg}$ ) or change in conscious state. ordered). The PACT also contains information regarding oxygen therapy, analgesia administered in PACU and related charts specific to the patient and surgical procedure. As an aid to nurses, the PACT includes a clinical handover checklist, adapted from an existing tool for standardising communication. ${ }^{20}$ The acronym, ISOBAR summarises the components of the checklist (Identification, Situation, Observation, Background, Assessment and Request or Recommendation).

\section{Data collection}

Data will be collected prospectively in PACU on the day of surgery and retrospectively following hospital discharge, from the following three sources.

\section{In PACU data collection}

Data will be collected by a research nurse, observing nursing care from the time of a patient's admission to PACU until handover to the ward nurse, using the 'Tap Forms' (2013 Tap Zapp Software Inc) application on iPads, with the collated data from 'Tap forms' exported into Microsoft Excel for validation. The data to be collected includes patient demographics, procedural data (surgical procedure, length of stay in PACU), and nursing assessment of patient readiness for discharge from PACU, nursing handover from PACU to ward nurse, patient outcomes (PACU discharge destination, any complications and adverse events in PACU).

\section{Medical record audit}

A subsequent audit of each patient's medical record after hospital discharge will be conducted to confirm the data collected in PACU and to provide additional data such as: anaesthetic type, anaesthetic agent used, the American Society of Anesthesiologists (ASA) Score (a global score that assesses the physical status of patients before surgery), assessment of any complications or adverse events that may have occurred during hospital stay, length of hospital admission, discharge destination from hospital and in-hospital mortality.

Fidelity measures to assess the extent to which the PACT is used appropriately in PACU will include adherence to the content of the tool, frequency of use, time taken for completion and coverage across different times of day and days of the week. The compliance rate will also be assessed by monitoring a random sample of PACU discharge documents from before and after the intervention to determine the percentage of items that have been completed. The use of the PACT by nurses caring for patients in PACU will be observed and facilitators and barriers to its use will be identified through direct observation.

\section{Economic evaluation}

The study will include a cost-effectiveness analysis. The evaluation will be conducted from a third party payer perspective to examine the costs and benefits for the use of the PACT. The evaluation will use the rate of 
complications and adverse events, mortality and length of stay as the outcome measures. The cost of admission to hospital will be determined through the use of Australian Refined Diagnosis Related Groups (AR-DRGs) and the Nationally Efficient Price for commonwealth funded public hospital services. ${ }^{21}$ The results of the analysis will be reported as a series of incremental cost-effectiveness ratios. The $95 \%$ CIs for the outcome measures will be used in sensitivity and uncertainty analysis.

\section{Outcomes}

The primary outcomes under investigation are the rate of complications, adverse events and mortality, the length of stay in PACU and in hospital. Complications and serious adverse events that will be reported are shown in box 1 and include clinical deterioration, prolonged stay in PACU, unplanned return to theatre, unplanned admission to ICU or readmission to hospital. Complications were defined as any deviation from the normal post-operative course. ${ }^{22}$ The frequency of complications and adverse events will be reported as the number per 100 patients, and per category, along with the proportion of patients with one or more complication or adverse event. Common Terminology Criteria for Adverse Events (CTCAE) V.4.0 $0^{23}$ will be used to assess the severity of adverse events.

The secondary outcomes are the costs incurred by the health service, clinical reliability and fidelity measures of the PACT, observation of nursing handover to ward staff, duration of nursing handover and the identification of any deficits in the PACT. These outcomes will be measured through observation, medical record audit and from health service data sets, as detailed above. Phase 1 data collection in PACU has been scheduled for June to October in 2012. The PACT was to be implemented in March 2014 and Phase 2 data collection in PACU take place during July-September 2014. Medical record audit is to be completed following the in-PACU data collection for each phase. Economic data will be available from the health service after coding has been completed, in December 2014.

\section{Statistical analysis}

The $\mathrm{CMH}$ test will be used to compare the proportions of adverse events and mortality between the two groups (pre-intervention and post-intervention groups). The CMH test takes account of the hospital clustering effect and allows for variation between the strata in the underlying rates. The common OR and its $95 \%$ CI will also be reported as well as the results of the Breslow-Day test for homogeneity of the ORs across the strata. If there is significant heterogeneity in the ORs, the groups will also be compared, using $\chi^{2}$ tests, in three separate subset analyses-one for each of the hospitals. In supportive analyses, generalised linear mixed models (GLMMs) will be used to compare the rates of adverse events and mortality after adjusting for baseline measurements at patient and/or hospital level. Analogous linear mixed models will be used to analyse the continuous-scale secondary end points. A series of exploratory analyses on subgroups and the impact of covariates, on estimates of the effect of the intervention, will also be examined. Length of time in PACU, length of handover and length of hospital stay will be considered as time to event data. Survival rates will be calculated and illustrated by the Kaplan-Meier method and further analysed by the log-rank test for univariate analysis (stratified by hospitals). Variables that reveal prognostic or effect modifying potential on the outcome as suggested by univariate analysis will be subsequently evaluated by the proportional Cox regression for multivariate analysis. HRs with the corresponding $95 \%$ CIs will be reported. $p$ Values $<0.05$ were considered statistically significant. Data will be analysed using Stata V.13 or later (StataCorp., College Station, Texas: StataCorp LP.).

\section{Dissemination}

A waiver of consent was granted as the study was assessed as low risk with no patient-related data collected other than that which is required for patient care, and contained within the medical record. During in-PACU data collection, the research nurse will be instructed to observe patient care and not to approach patients, or impact on nursing care. Patient identifiers will only be used during data collection and once the data has been validated, these will be removed from the data set prior to analysis. PACU managers and nursing staff will be informed of the study, and verbal consent to participate will be obtained from all the nurses observed in PACU.

The findings of the study will be disseminated through a report to the funding body, consultation and presentation to the clinicians and executives of the health service, conference presentations, publications in peer-reviewed journals as well as being deposited in an institutional repository, Deakin Research Online.

\section{DISCUSSION}

Post-operative complications are relatively common, occurring in between $3 \%$ and $17 \%$ of patients admitted for surgery. ${ }^{5} 924$ Complications are associated with increased costs and prolonged length of stay, even after adjusting for type of surgery and patient comorbid conditions.8 925 Patients who experience post-operative complications consume considerably more healthcare resources than patients whose surgical admission is without any adverse events. Reported post-operative complication rates are that for every 100 patients, 8 will develop an infection (such as pneumonia or surgical site sepsis), 3 will require intervention to relieve respiratory distress; 2 will require intervention for cardiovascular reasons and 2 will experience excessive bleeding. ${ }^{7}$

The evidence-based discharge criteria that were used in the PACT were identified from the findings of a systematic review of the literature previously conducted by the 
researchers and an extensive process of stakeholder consultation with expert nurses, anaesthetists and other postoperative healthcare professionals. In summary this project will examine whether the use of the PACT results in improved patient outcomes and reduced healthcare costs.

\section{Potential limitations of the study}

The use of a non-randomised research design, with historical control group, does not allow for causality to be determined. This design was chosen because the health service had already decided to standardise documentation and assessment in PACU, with the changes being implemented in all three hospitals at the same time. The data collection during the study will occur in two different time periods, before and after the introduction of the PACT. There may be external factors which increase or decrease surgical risk between these two periods. However, this design is practical and effective and has been used previously to demonstrate the benefit of a surgical safety checklist. ${ }^{7}$ An analysis will be undertaken to determine whether there were any statistically significant differences in the two groups prior to surgery, in terms of their age, gender, surgical risk, the presence of comorbidities and the number and types of surgical procedures. Where relevant, these potential confounders will be adjusted for in the final analysis. Further studies using randomised research designs will be required to determine if the differences observed in study outcomes can be causally attributed to the use of the PACT.

\section{Potential healthcare policy impact of the study}

This evaluation of the use of PACT, for nursing assessment of patient readiness for discharge from PACU following surgery, will have clinical relevance and impact on healthcare policy. Health service providers constantly review the policies and procedures relating to healthcare provision within their organisations. The changes made to the Post-anaesthetic Care Record have been substantial and further revision may be required. However, the findings of this study will add to the evidence base for clinicians and decision-makers about caring for patients in PACU.

\section{CONCLUSION}

Nursing assessment of patients in PACU to determine their readiness for discharge using the PACT has the potential to benefit all adult patients undergoing surgery. Early recognition and response to patient deterioration may result in improved patient outcomes and fewer serious adverse events following surgery. The associated improved health outcomes for the patient could also help to reduce the costs to the healthcare network.

\section{Author affiliations}

${ }^{1}$ School of Nursing and Midwifery, Deakin University, Burwood, Victoria, Australia

${ }^{2}$ Eastern Health-Deakin University Nursing \& Midwifery Research Centre, Box Hill, Victoria, Australia
${ }^{3}$ School of Nursing and Midwifery, Plymouth University, Plymouth, Devon, UK ${ }^{4}$ Department of Deakin Health Economics, Deakin University, Burwood, Victoria, Australia

${ }^{5}$ Biostatistics Unit, Faculty of Health, Deakin University, Burwood, Victoria, Australia

Acknowledgements The authors gratefully acknowledge the expert advice of clinicians at the health service, especially PACU nurse unit managers, with respect to patient assessment and nursing practice in post-anaesthetic care.

Contributors All the authors made substantial contributions to the conception and design of this study protocol. MS undertook primary responsibility for the acquisition of funding and establishing a steering committee to oversee the project. MS and NMP will be responsible for the general supervision of the research nurses collecting the data, while SC is responsible for obtaining the economic and cost data. MM contributed to determining sample size and the plan for statistical analysis. NMP and BK will advise the research team with interpretation of clinical data, especially with respect to complications and adverse events. All the authors have contributed to the revising of the content and have read and approved the final manuscript.

Funding This work was supported by Health Contributions Fund (HCF) Health \& Medical Research Foundation, grant number MSDU1008201158-20120118.

Competing interests None declared.

Ethics approval This study has been approved by the Eastern Health Human Research and Ethics Committee and by the Deakin University Human Research Ethics Committee.

Provenance and peer review Not commissioned; externally peer reviewed.

Open Access This is an Open Access article distributed in accordance with the Creative Commons Attribution Non Commercial (CC BY-NC 4.0) license, which permits others to distribute, remix, adapt, build upon this work noncommercially, and license their derivative works on different terms, provided the original work is properly cited and the use is non-commercial. See: http:// creativecommons.org/licenses/by-nc/4.0/

\section{REFERENCES}

1. Weiser TG, Regenbogen SE, Thompson KD, et al. An estimation of the global volume of surgery: a modelling strategy based on available data. Lancet 2008;372:139-44.

2. Brennan TA, Leape LL, Laird NM, et al. Incidence of adverse events and negligence in hospitalized patients: results of the Harvard Medical Practice Study I. 1991. BMJ Qual Saf 2004;13:145-51.

3. Gawande AA, Thomas EJ, Zinner MJ, et al. The incidence and nature of surgical adverse events in Colorado and Utah in 1992. Surgery 1999;126:66-75.

4. Kable AK, Gibberd RW, Spiegelman AD. Adverse events in surgical patients in Australia. Int J Qual Health Care 2002;14:269-76.

5. Bosma E, Veen EJ, Roukema JA. Incidence, nature and impact of error in surgery. Br J Surg 2011;98:1654-9.

6. de Vries EN, Ramrattan MA, Smorenburg SM, et al. The incidence and nature of in-hospital adverse events: a systematic review. BMJ Qual Saf 2008;17:216-23.

7. de Vries EN, Prins HA, Crolla R, et al. Effect of a comprehensive surgical safety system on patient outcomes. $N$ Engl J Med 2010;363:1928-37.

8. Birkmeyer JD, Gust C, Dimick JB, et al. Hospital quality and the cost of inpatient surgery in the United States. Ann Surg 2012;255:1-5.

9. Khan NA, Quan H, Bugar JM, et al. Association of postoperative complications with hospital costs and length of stay in a tertiary care center. J Gen Intern Med 2006;21:177-80.

10. Prowse MA, Lyne PA. Clinical effectiveness in the post-anaesthesia care unit: how nursing knowledge contributes to achieving intended patient outcomes. J Adv Nurs 2000;31:1115-24.

11. Braaf S, Manias E, Riley R. The role of documents and documentation in communication failure across the perioperative pathway. A literature review. Int J Nurs Stud 2011;48:1024-38.

12. Swatton S. A discharge protocol for the postanaesthetic recovery unit. Br J Perioper Nurs 2004;14:74-80. 
13. Aldrete JA. Modifications to the postanesthesia score for use in ambulatory surgery. $J$ Perianesth Nurs 1998; 13:148-55.

14. Aldrete JA. Post-anesthetic recovery score. J Am Coll Surg 2007;205:e3

15. Phillips NM, Street M, Kent B, et al. Post-anaesthetic discharge scoring criteria: key findings from a systematic review. Int J Evid Based Healthc 2013:11:275-84

16. Phillips NM, Haesler E, Street M, et al. Post-anaesthetic discharge scoring criteria: a comprehensive systematic review. JBI Library Syst Rev 2011;9(41):1679-713.

17. Phillips NM, Street M, Kent B, et al. Determining criteria to assess patient readiness for discharge from postanaesthetic care: an international Delphi study. J Clin Nurs 2014;23:3345-55.

18. Patient safety First. Reducing harm in perioperative care. http://www. patientsafetyfirst.nhs.uk/Content.aspx?path=/interventions/ Perioperativecare (accessed 30 Oct 2014).

19. Zegers M, de Bruijne MC, de Keizer B, et al. The incidence, root-causes, and outcomes of adverse events in surgical units: implication for potential prevention strategies. Patient Saf Surg 2011:5:13.
20. Porteous J, Stewart-Wynne EG, Connolly M, et al. iSoBAR-a concept and handover checklist: the National Clinical Handover Initiative. Med J Aust 2009;190:152.

21. Independent Hospital Pricing Authority (IHPA) Costing 2014. http:// www.ihpa.gov.au/internet/ihpa/publishing.nst/Content/costing-lp (accessed 30 Oct 2014).

22. Dindo D, Demartines N, Clavien PA. Classification of surgical complications: a new proposal with evaluation in a cohort of 6336 patients and results of a survey. Ann Surg 2004;240:205

23. U.S.Department of Health and Human Services, National Institutes of Health, National Cancer Institute. Common Terminology Criteria for Adverse Events (CTCAE) Version 4.03, 2010. http://evs.nci.nih. gov/ftp1/CTCAE/CTCAE_4.03_2010-06-14_QuickReference_5×7. pdf (accessed 2 Mar 2015)

24. Dimick JB, Chen SL, Taheri PA, et al. Hospital costs associated with surgical complications: a report from the private-sector National Surgical Quality Improvement Program. J Am Coll Surg 2004;199:531

25. Dimick JB, Weeks WB, Karia RJ, et al. Who pays for poor surgical quality? Building a business case for quality improvement. J Am Coll Surg 2006;202:933-7. 